\title{
Vital Signs: Clinical Characteristics of Patients with Confirmed Acute Flaccid Myelitis, United States, 2018
} Sarah Kidd, $\mathrm{MD}^{1}$; Adriana Lopez, MHS ${ }^{1}$; W. Allan Nix ${ }^{1}$; Gloria Anyalechi, MD²; Megumi Itoh, MD ${ }^{3}$; Eileen Yee, MD ${ }^{1}$;
M. Steven Oberste, $\mathrm{PhD}^{1}$; Janell Routh, MD

On August 4, 2020, this report was posted as an MMWR Early Release on the MMWR website (https://www.cdc.gov/mmwr).

\section{Abstract}

Background: Acute flaccid myelitis (AFM) is a serious neurologic syndrome that affects mostly children and is characterized by the acute onset of limb weakness or paralysis. Since U.S. surveillance for AFM began in 2014, reported cases have peaked biennially. This report describes the clinical characteristics of AFM patients during 2018, the most recent peak year.

Methods: Medical records from persons meeting AFM clinical criterion (acute onset of flaccid limb weakness) were submitted to CDC. Patients with confirmed AFM met the clinical criterion and had magnetic resonance imaging indicating spinal cord lesions largely restricted to gray matter and spanning one or more vertebral segments. Symptoms, physical findings, test and imaging results, and hospitalization data were abstracted and described.

Results: Among 238 patients with confirmed AFM during 2018, median age was 5.3 years. Among the 238 patients, 205 (86\%) had onset during August-November. Most (92\%) had prodromal fever, respiratory illness, or both beginning a median of 6 days before weakness onset. In addition to weakness, common symptoms at clinical evaluation were gait difficulty (52\%), neck or back pain (47\%), fever (35\%), and limb pain (34\%). Among 211 who were outpatients when weakness began, most (76\%) sought medical care within 1 day, and $64 \%$ first sought treatment at an emergency department. Overall, $98 \%$ of patients were hospitalized, 54\% were admitted to an intensive care unit, and $23 \%$ required endotracheal intubation and mechanical ventilation.

Conclusion: Clinicians should suspect AFM in children with acute flaccid limb weakness, especially during August-November and when accompanied by neck or back pain and a recent history of febrile respiratory illness. Increasing awareness in frontline settings such as emergency departments should aid rapid recognition and hospitalization for AFM.

\section{Introduction}

Acute flaccid myelitis (AFM) is a serious neurologic syndrome that can cause paralysis, predominantly in previously healthy children. Similar to poliomyelitis-associated acute flaccid paralysis caused by poliovirus infection, AFM is a syndrome characterized by the acute onset of flaccid limb weakness accompanied by predominantly gray matter lesions in the spinal cord. AFM can progress rapidly over the course of hours or days, leading to permanent paralysis and the lifethreatening complication of respiratory failure (1).

National surveillance for AFM was initiated in 2014, after California and Colorado reported clusters of AFM or acute limb weakness among previously healthy children, none of whom had laboratory or epidemiologic evidence of poliovirus infection $(2,3)$. Since 2014, reported AFM cases have peaked in the late summer to early fall every 2 years in the United States (4). Although national case reporting for AFM did not begin until 2014, retrospective case investigations have documented sporadic cases before 2014 and increased numbers of cases during 2014, 2016, and 2018 (5,6). Together, these data suggest that the epidemiology of AFM shifted during or shortly before 2014, and likely reflect a new or emerging etiology.

Multiple viruses, including West Nile virus, adenovirus, and nonpolio enteroviruses, are known to cause AFM in a small percentage of infected persons $(7-10)$. Pathogens are rarely recovered from the cerebrospinal fluid (CSF) of AFM patients, but enteroviruses are the most common pathogens detected in nonsterile site specimens, such as respiratory and stool specimens $(4,11)$. Enterovirus D68 (EV-D68) is the most common enterovirus type identified among AFM patients; poliovirus has not been detected in any cases $(4,11,12)$. In addition, recent data, including animal model studies and studies of enterovirus-binding antibodies in CSF, indicate that nonpolio enteroviruses, and EV-D68 in particular, are likely a primary cause of AFM in the United States since 2014 (13-17). However, other viruses that cause AFM might be contributing to the biennial peaks. A cluster of 11 AFM cases in Colorado associated with enterovirus A71 (EV-A71) contributed to the number of cases reported in $2018(18,19)$. 


\section{Summary}

What is already known about this topic?

Since U.S. surveillance for acute flaccid myelitis (AFM) began in 2014, reported cases have peaked biennially. Most cases occur in children during late summer and early fall.

What is added by this report?

Among 238 patients with confirmed AFM during 2018, most (92\%) had prodromal fever, respiratory illness, or both. In addition to weakness, common symptoms were gait difficulty (52\%), neck or back pain (47\%), fever (35\%), and limb pain (34\%). Among 211 who were outpatients when weakness began, $64 \%$ sought treatment at an emergency department. Overall, $23 \%$ required endotracheal intubation and mechanical ventilation.

What are the implications for public health practice?

Clinicians should suspect AFM in children with acute flaccid limb weakness, especially when accompanied by neck or back pain and a recent history of febrile respiratory illness. Increasing awareness in frontline settings such as emergency departments should aid rapid recognition and hospitalization for AFM.

Based on the observed biennial pattern, another increase in AFM cases is anticipated to occur in the United States in late summer/early fall 2020 (https://www.cdc.gov/grand-rounds/ pp/2020/20200703-acute-flaccid-myelitis.html). This report summarizes findings from review of medical records from patients with confirmed AFM in 2018, including cases known to be associated with EV-D68 and EV-A71, and describes the clinical characteristics of patients and settings and timing of seeking medical care for limb weakness. These data might facilitate rapid case recognition of AFM and prompt referral to care.

\section{Methods}

As part of national surveillance, health departments report cases meeting the clinical criterion for AFM (acute onset of flaccid limb weakness) to CDC, along with a patient summary form (completed by health departments) and relevant components of patient medical records when available, including admission and discharge notes, neurology and infectious disease consult notes, laboratory reports, and brain and spine magnetic resonance imaging (MRI) reports. A confirmed AFM case was defined as an illness meeting the clinical criterion with an MRI indicating a spinal cord lesion largely restricted to gray matter and spanning one or more vertebral segments. Confirmed cases were described by month of onset for August 2014-June 2020. In addition, data on the clinical evaluation and hospitalization for limb weakness, including prodromal and initial symptoms at evaluation, neurologic exam findings, laboratory results, MRI findings, and type of medical setting where the patient was evaluated, were abstracted from records of patients with confirmed AFM who had onset of limb weakness during 2018.

Neurologic exam findings were abstracted from the first neurology consultation note following onset of limb weakness or, if that was not available, from the first and most complete documented neurologic exam following the onset of weakness. MRI findings were abstracted from reports of the most abnormal brain and spine MRIs available.

Abstracted laboratory findings included results for enterovirus/rhinovirus $(\mathrm{EV} / \mathrm{RV})^{*}$ detection and typing tests performed at external laboratories or CDC's AFM laboratory. CDC laboratory methods have been described previously (4). EV-D68 and EV-A71 cases were defined as a confirmed AFM case with a CSF, respiratory, stool, or serum specimen that tested positive for EV-D68 or EV-A71, respectively, at either an external laboratory or the CDC laboratory. Data on long-term patient outcomes were not available for this analysis.

\section{Results}

Since surveillance for AFM began following the initial clusters reported in 2014, nationwide outbreaks have occurred in 2016 and 2018 (Figure). A total of 238 confirmed AFM cases with onset in 2018 were reported to CDC; among these, onset of limb weakness in 205 (86\%) occurred during AugustNovember, including 87 (37\%) with onset during September (Figure). Among 219 (92\%) patients receiving tests for EV/RV, 107 (49\%) had at least one EV/RV-positive specimen (Table 1). A higher proportion of respiratory specimens were positive for EV/RV (48\%), compared with other specimen sources (3\% [serum] to 20\% [stool]). EV-D68 was the most common virus type identified, and most EV-D68 (33 of 34) and EV-A71 (10 of 12) cases were identified from respiratory specimens.

The median age of patients with confirmed AFM was 5.3 years (range $=0.5-81.8$ years), and $58 \%$ were male (Table 2). Patients were reported from 42 states throughout the country; $53 \%$ identified as white, $20 \%$ as Hispanic, and $9 \%$ as black. A prodromal illness preceding the onset of limb weakness was documented in most (97\%) patients, with respiratory illness $(80 \%)$ and fever $(77 \%)$ the most commonly reported prodromal signs and symptoms. Many patients also had documented neck or back pain $(46 \%)$ or headache $(37 \%)$ preceding the onset of limb weakness. Among those who reported prodromal symptoms, the onset of respiratory illness generally occurred earlier than the onset of fever, headache, or neck or back pain.

\footnotetext{
*Enteroviruses and rhinoviruses are closely related picornaviruses. Most widely available polymerase chain reaction tests for enterovirus amplify a viral gene sequence that is highly conserved and is similar between enteroviruses and rhinoviruses. Therefore, these tests do not distinguish between enteroviruses or rhinoviruses, and additional testing is needed to differentiate between these groups of viruses.
} 
FIGURE. Confirmed cases of acute flaccid myelitis (AFM) reported to CDC ( $=633$ ), by month and year of onset - United States, August 1, 2014-June 30, 2020*,†

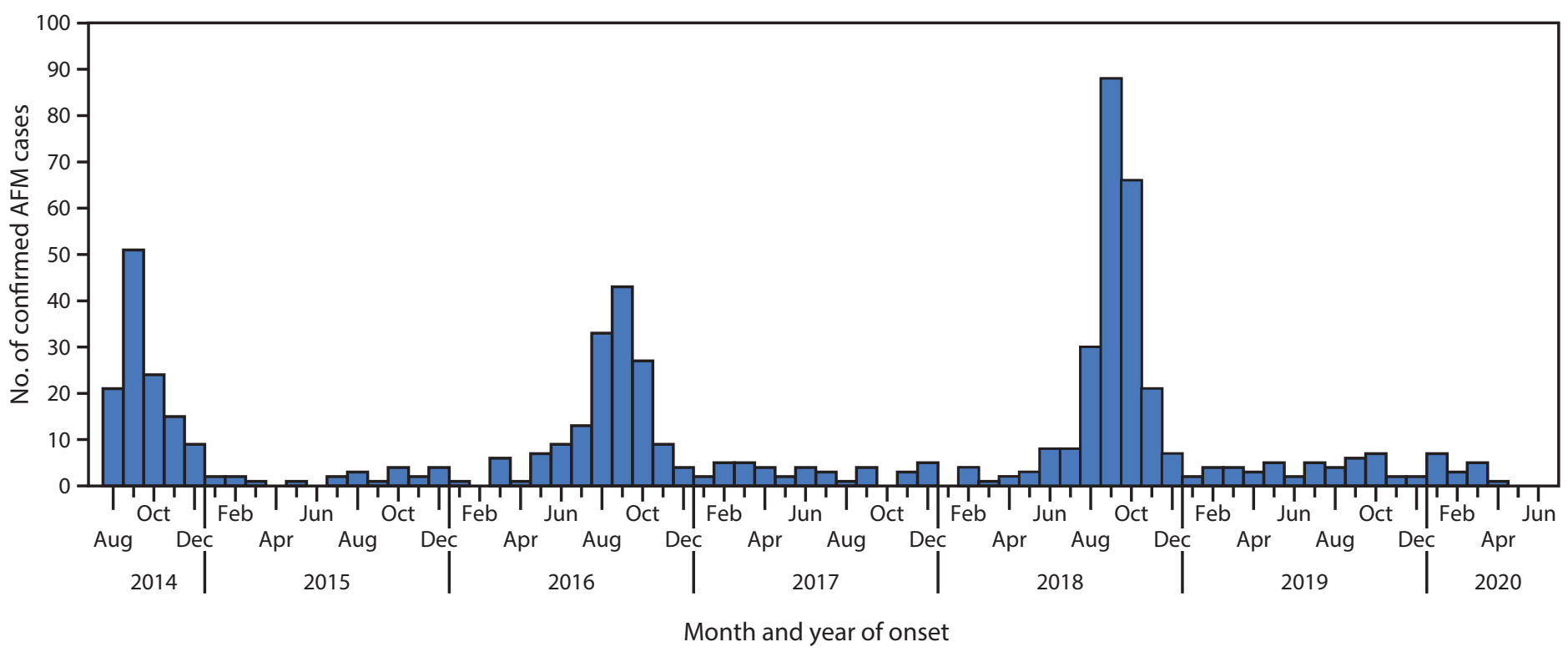

* As of July 24, 2020.

${ }^{\dagger}$ Total cases reported each year: $2014=120 ; 2015=22 ; 2016=153 ; 2017=38 ; 2018=238 ; 2019=46 ; 2020=16$.

At the time that patients were seen at a hospital for weakness, the most commonly documented symptoms were gait difficulty $(52 \%)$, neck or back pain (47\%), fever $(35 \%)$, and pain in the affected limb or limbs (34\%). Upper extremity weakness (64\%) was more commonly noted on initial neurologic exam than was lower extremity weakness (36\%). Only $13 \%$ of patients had documented sensory abnormalities; $21 \%$ had cranial nerve abnormalities, and $5 \%$ had altered mental status on exam.

All patients with confirmed AFM had, by definition, at least one abnormal spinal cord MRI indicating predominantly gray matter lesions. Based on medical records received at CDC, 227 (95\%) patients had a brain MRI, 233 (98\%) had a cervical spine MRI, 205 (86\%) had a thoracic spine MRI, and 177 (74\%) had a lumbosacral spine MRI performed. Among those receiving MRIs, cervical cord lesions were most commonly observed (219 of 233 ; 94\%), followed by thoracic cord lesions ( 176 of 205; 86\%). Although fewer patients had a lumbosacral spine MRI, conus lesions were seen in 40\% (71 of 177) of those with MRIs. Brainstem lesions were observed in $44 \%$ (100 of 227) of those who received a brain MRI.

Most $(233$ of 238 ; 98\%) patients were hospitalized (Table 3). Twenty-five patients (11\%) were hospitalized at least 1 day before onset of limb weakness and developed weakness while inpatients, $206(87 \%)$ were hospitalized on or after the day limb weakness began, and the date of onset for two patients was unclear. Overall, $54 \%$ of all patients were admitted to an intensive care unit, and $23 \%$ required intubation and mechanical ventilation. Steroids and intravenous immunoglobulin (IVIG) were the commonly administered treatments (Table 3).
Among the 211 patients who developed limb weakness as an outpatient (including five who were never hospitalized), most (134 of $211 ; 64 \%$ ) initially sought treatment at an emergency department and $49(23 \%)$ at a primary care provider. Most (160 of $211 ; 76 \%$ ) sought medical care within 1 day of onset of limb weakness. Similarly, among patients hospitalized on the same day or after onset of weakness, most (134 of 206; $65 \%$ ) were hospitalized within 1 day of onset.

When EV-D68 and EV-A71 cases were analyzed, patients with known EV-D68 infection were older (median age $=5.9$ years) than were those with EV-A71 infection (median age $=1.6$ years). In addition, patients with EV-D68 were reported from across the country, whereas 11 of 12 patients with EV-A71 were geographically and temporally clustered in Colorado (Table 2). All EV-D68 and EV-A71 cases had a prodromal illness before onset of limb weakness. Prodromal respiratory illness was more common among EV-D68 (97\%) than among EV-A71 cases (58\%). Prodromal rash was more common among EV-A71 (58\%) than among EV-D68 cases (9\%). The most common signs and symptoms accompanying limb weakness among EV-D68 cases were neck or back pain (59\%), gait difficulty (56\%), and fever (47\%), whereas among EV-A71 cases, the most common signs and symptoms were fever (67\%), ataxia (67\%), gait difficulty (50\%), and altered consciousness (50\%). Abnormal findings on brain MRI were less common among EV-D68 than among EV-A71 cases, but a higher proportion of EV-D68 than EV-A71 cases were admitted to an intensive care unit and required intubation and mechanical ventilation (Table 3). 
TABLE 1. Enterovirus/rhinovirus (EV/RV) polymerase chain reaction test results from respiratory, stool, cerebrospinal fluid (CSF), and serum specimens collected from patients with onset of confirmed acute flaccid myelitis $(N=238)$ - United States, 2018

\begin{tabular}{|c|c|c|c|}
\hline Specimen source & $\begin{array}{c}\text { No. of patients with specimens } \\
\text { available } \\
\text { (\% of } 238 \text { ) }\end{array}$ & No. (\%) positive & EV/RV type results (no.) \\
\hline Any source* & $219(92)$ & $107(49)$ & $\begin{array}{l}\text { EV-D68 (34) } \\
\text { EV-A71 (12) } \\
\text { Coxsackievirus A2 (1) } \\
\text { Coxsackievirus A4 (1) } \\
\text { Coxsackievirus A9 (1) } \\
\text { Coxsackievirus A16 (1) } \\
\text { Coxsackievirus B3 (1) } \\
\text { Echovirus 11 (1) } \\
\text { RV-A101 (2) } \\
\text { RV-A24 (1) } \\
\text { RV-A38 (1) } \\
\text { RV-A54 (1) } \\
\text { RV-A81 (1) } \\
\text { RV-A85 (1) } \\
\text { RV-B4 (1) } \\
\text { RV-C54 (1) } \\
\text { Other/Untyped EV/RV (46) }\end{array}$ \\
\hline Respiratory & $190(80)$ & $92(48)$ & $\begin{array}{l}\text { EV-D68 (33) } \\
\text { EV-A71 (10) } \\
\text { RV-A101 (2) } \\
\text { RV-A24 (1) } \\
\text { RV-A38 (1) } \\
\text { RV-A54 (1) } \\
\text { RV-A81 (1) } \\
\text { RV-A85 (1) } \\
\text { RV-B4 (1) } \\
\text { RV-C54 (1) } \\
\text { Other/Untyped EV/RV (40) }\end{array}$ \\
\hline Stool & $119(50)$ & $24(20)$ & $\begin{array}{l}\text { EV-D68 (3) } \\
\text { EV-A71 (2) } \\
\text { Coxsackievirus A2 (1) } \\
\text { Coxsackievirus A4 (1) } \\
\text { Coxsackievirus A9 (1) } \\
\text { Coxsackievirus A16 (1) } \\
\text { Coxsackievirus B3 (1) } \\
\text { Echovirus } 11 \text { (1) } \\
\text { Other/Untyped EV/RV (13) }\end{array}$ \\
\hline CSF & $187(79)$ & $9(5)$ & $\begin{array}{l}\text { EV-D68 (2) } \\
\text { EV-A71 (1) } \\
\text { Other/Untyped EV/RV (6) }\end{array}$ \\
\hline Serum & $90(38)$ & $3(3)$ & $\begin{array}{l}\text { EV-D68 (1) } \\
\text { Echovirus } 11 \text { (1) } \\
\text { Other/Untyped EV/RV (1) }\end{array}$ \\
\hline
\end{tabular}

* Some patients had multiple positive specimens.

\section{Discussion}

The findings in this report are consistent with, and build upon, previous reports describing patients with confirmed AFM during 2018 and earlier peak years $(4,11,12,19)$. The current analysis supports previous reports documenting the frequent presence of respiratory symptoms, fever, or both before the onset of limb weakness and a predominance of upper limb involvement among AFM patients. In addition, prodromal neck or back pain or headache before onset of limb weakness were identified and described in the present cohort. Eleven percent of AFM patients were hospitalized at least 1 day before the onset of limb weakness, indicating that prodromal symptoms might be severe in some patients.
Evaluating a child with weakness and differentiating AFM from other causes of weakness can be challenging. In younger children especially, weakness might manifest as decreased use of a limb, which might mistakenly be attributed to musculoskeletal pain or injury. Gait difficulty, neck or back pain, fever, limb pain, and headache were often present when AFM patients sought care for limb weakness. The presence of these or any neurologic signs or symptoms in a child with acute limb weakness or decreased use of an extremity should heighten clinical suspicion of AFM, particularly in the setting of a recent respiratory or febrile illness. 
TABLE 2. Demographic and clinical characteristics of patients with onset of confirmed acute flaccid myelitis ( $\mathrm{N}=238$ ), by virus type United States, 2018

\begin{tabular}{|c|c|c|c|c|}
\hline \multirow[b]{2}{*}{ Characteristic } & \multicolumn{4}{|c|}{ No. (\%) } \\
\hline & $\begin{array}{l}\text { EV-D68 } \\
(n=34)\end{array}$ & $\begin{array}{l}\text { EV-A71 } \\
(n=12)\end{array}$ & $\begin{array}{c}\text { Other } \\
(n=192)^{*}\end{array}$ & $\begin{array}{c}\text { Total } \\
(\mathrm{N}=238)\end{array}$ \\
\hline \multicolumn{5}{|l|}{ Demographic } \\
\hline Median age, yrs & 5.9 & 1.6 & 5.3 & 5.3 \\
\hline Range & $1.4-56.9$ & $0.9-32.7$ & $0.5-81.8$ & $0.5-81.8$ \\
\hline IQR & 3.7-7.9 & $1.1-2.1$ & $3.4-8.3$ & $3.3-8.2$ \\
\hline Male sex & $20(59)$ & $11(92)$ & $107(56)$ & $138(58)$ \\
\hline \multicolumn{5}{|l|}{ Geographic region } \\
\hline South & $16(47)$ & $0(0)$ & $64(33)$ & $80(34)$ \\
\hline Midwest & $6(18)$ & $1(8)$ & $54(28)$ & $61(26)$ \\
\hline West & $6(18)$ & $11(92)$ & $39(20)$ & $56(24)$ \\
\hline Northeast & $6(18)$ & $0(0)$ & $35(18)$ & $41(17)$ \\
\hline \multicolumn{5}{|l|}{ Race/Ethnicity } \\
\hline White & $23(68)$ & $10(83)$ & $92(48)$ & $125(53)$ \\
\hline Hispanic & $5(15)$ & $1(8)$ & $41(21)$ & $47(20)$ \\
\hline Black & $2(6)$ & $0(0)$ & $19(10)$ & $21(9)$ \\
\hline Asian & $1(3)$ & $0(0)$ & $7(4)$ & $8(3)$ \\
\hline Multiracial & $0(0)$ & $0(0)$ & $4(2)$ & $4(2)$ \\
\hline Native Hawaiian/Pacific Islander & $0(0)$ & $0(0)$ & $1(1)$ & $1(0)$ \\
\hline Unknown & $3(9)$ & $1(8)$ & $28(15)$ & $32(13)$ \\
\hline \multicolumn{5}{|c|}{ Prodromal signs/symptoms in the 4 weeks before onset of limb weakness } \\
\hline Any illness, no. (\% of total) & $34(100)$ & $12(100)$ & $184(96)$ & $230(97)$ \\
\hline Days before weakness onset $(\mathrm{IQR})^{\dagger}$ & $5(4-7)$ & $3(2.5-6.5)$ & $6(3-9)$ & $6(3-9)$ \\
\hline Any respiratory illness or fever, no. (\% of total) & $34(100)$ & $12(100)$ & $174(91)$ & $220(92)$ \\
\hline Days before weakness onset $(\mathrm{IQR})^{\dagger}$ & $5(4-7)$ & $3(2-5)$ & $6(3-9)$ & $6(3-8)$ \\
\hline Any respiratory illness, no. (\% of total) & $33(97)$ & $7(58)$ & $151(79)$ & $191(80)$ \\
\hline Days before weakness onset $(\mathrm{IQR})^{\dagger}$ & $5(4-7)$ & $3(1-5)$ & $6(4-9)$ & $6(4-9)$ \\
\hline Any fever, no. (\% of total) & $28(82)$ & $12(100)$ & $144(75)$ & $184(77)$ \\
\hline Days before weakness onset $(\mathrm{IQR})^{\dagger}$ & $3(2-6)$ & $3(2-5)$ & $3(2-6)$ & $3(2-6)$ \\
\hline Neck/back pain, no. (\% of total) & $18(53)$ & $4(33)$ & $88(46)$ & $110(46)$ \\
\hline Days before weakness onset $(\mathrm{IQR})^{\dagger}$ & $2(1-3)$ & $1.5(0.5-4)$ & $1(1-3)$ & $1.5(1-3)$ \\
\hline Headache, no. (\% of total) & $12(35)$ & $2(17)$ & $73(38)$ & $87(37)$ \\
\hline Days before weakness onset (IQR) ${ }^{\dagger}$ & $3(2-3)$ & $2(2-2)$ & $2(1-6)$ & $2(1-5.5)$ \\
\hline Any gastrointestinal illness, no. (\% of total) & $9(26)$ & $6(50)$ & $38(20)$ & $53(22)$ \\
\hline Days before weakness onset $(\mathrm{IQR})^{\dagger}$ & $2(1-4)$ & $2.5(2-3)$ & $3(2-4)$ & $2(2-4)$ \\
\hline Rash, no. (\% of total) & $3(9)$ & $7(58)$ & $13(7)$ & $23(10)$ \\
\hline Days before weakness onset $(I Q R)^{\dagger}$ & $4.5(2-7)$ & $4(3-6)$ & $9.5(3-18.5)$ & $4(3-7)$ \\
\hline \multicolumn{5}{|l|}{ Signs/Symptoms at evaluation } \\
\hline Gait difficulty & $19(56)$ & $6(50)$ & $99(52)$ & $124(52)$ \\
\hline Pain in neck or back & $20(59)$ & $4(33)$ & $87(45)$ & $111(47)$ \\
\hline Fever & $16(47)$ & $8(67)$ & $59(31)$ & $83(35)$ \\
\hline Pain in affected limb(s) & $10(29)$ & $3(25)$ & $69(36)$ & $82(34)$ \\
\hline Headache & $13(38)$ & $2(17)$ & $51(27)$ & $66(28)$ \\
\hline Neck weakness & $7(21)$ & $1(8)$ & $31(16)$ & $39(16)$ \\
\hline Ataxia/Discoordination & $6(18)$ & $8(67)$ & $24(13)$ & $38(16)$ \\
\hline Facial weakness & $9(26)$ & $0(0)$ & $28(15)$ & $37(16)$ \\
\hline Dysphagia & $6(18)$ & $2(17)$ & $23(12)$ & $31(13)$ \\
\hline Bladder retention/incontinence & $2(6)$ & $3(25)$ & $23(12)$ & $28(12)$ \\
\hline Altered consciousness & $2(6)$ & $6(50)$ & $18(9)$ & $26(11)$ \\
\hline Dysarthria & $6(18)$ & $1(8)$ & $17(9)$ & $24(10)$ \\
\hline Numbness in affected limb(s) & $2(6)$ & $1(8)$ & $13(7)$ & $16(7)$ \\
\hline Paresthesia in affected limb(s) & $0(0)$ & $0(0)$ & $16(8)$ & $16(7)$ \\
\hline Bowel retention/incontinence & $1(3)$ & $3(25)$ & $10(5)$ & $14(6)$ \\
\hline Diplopia & $2(6)$ & $1(8)$ & $9(5)$ & $12(5)$ \\
\hline Ptosis & $3(9)$ & $0(0)$ & $6(3)$ & $9(4)$ \\
\hline Seizures & $2(6)$ & $1(8)$ & $3(2)$ & $6(3)$ \\
\hline \multicolumn{5}{|l|}{ Initial neurologic exam findings } \\
\hline Decreased strength in upper limb(s) & $22(65)$ & $4(33)$ & $127(66)$ & $153(64)$ \\
\hline Decreased strength in lower limb(s) & $16(47)$ & $2(17)$ & $67(35)$ & $85(36)$ \\
\hline Any sensory abnormalities & $5(15)$ & $0(0)$ & $25(13)$ & $30(13)$ \\
\hline Any cranial nerve abnormalities & $12(35)$ & $2(17)$ & 37 (19) & $51(21)$ \\
\hline Abnormal mental status & $1(3)$ & $5(42)$ & $6(3)$ & $12(5)$ \\
\hline
\end{tabular}

See table footnotes on next page. 
TABLE 2. (Continued) Demographic and clinical characteristics of patients with onset of confirmed acute flaccid myelitis ( $\mathrm{N}=238$ ), by virus type - United States, 2018

\begin{tabular}{|c|c|c|c|c|}
\hline \multirow[b]{2}{*}{ Characteristic } & \multicolumn{4}{|c|}{ No. (\%) } \\
\hline & $\begin{array}{l}\text { EV-D68 } \\
(n=34)\end{array}$ & $\begin{array}{l}\text { EV-A71 } \\
(n=12)\end{array}$ & $\begin{array}{c}\text { Other } \\
(n=192)^{*}\end{array}$ & $\begin{array}{c}\text { Total } \\
(\mathrm{N}=238)\end{array}$ \\
\hline \multicolumn{5}{|c|}{ CSF microscopic examination $\$$} \\
\hline CSF pleocytosis & $31 / 32(97)$ & $12 / 12(100)$ & 140/166 (84) & $183 / 210(87)$ \\
\hline Median cells $/ \mathrm{mm}^{3}$ & 95 & 125 & 91.5 & 94 \\
\hline Range & 9-499 & $17-685$ & $6-814$ & $6-814$ \\
\hline IQR & $36-170$ & $67-480$ & $42.5-157.5$ & $43-163$ \\
\hline \multicolumn{5}{|l|}{ MRI findings $s^{\pi}$} \\
\hline Supratentorial lesions & 3/33 (9) & $3 / 12(25)$ & 23/182 (13) & 29/227 (13) \\
\hline Brainstem lesions & $15 / 33(45)$ & $11 / 12(92)$ & 74/182 (41) & $100 / 227(44)$ \\
\hline Cerebellar lesions & $1 / 33(9)$ & 9/12 (75) & $38 / 182(21)$ & $50 / 227(22)$ \\
\hline Cervical cord lesions & $33 / 34(97)$ & $12 / 12(100)$ & $174 / 187(80)$ & $219 / 233(94)$ \\
\hline Thoracic cord lesions & $28 / 31(90)$ & 6/11 (55) & $142 / 163(87)$ & $176 / 205(86)$ \\
\hline Conus lesions & $16 / 25(64)$ & $2 / 5(40)$ & $2 / 5(36)$ & 71/177 (40) \\
\hline Nerve root enhancement & 5/33 (15) & $6 / 12(50)$ & 38/182 (21) & 49/227 (22) \\
\hline
\end{tabular}

Abbreviations: $\mathrm{CSF}=$ cerebrospinal fluid; $\mathrm{EV}=$ enterovirus; $\mathrm{IQR}=$ interquartile range; $\mathrm{MRI}=$ magnetic resonance imaging.

* Other category includes all patients who did not have a positive EV-D68 or EV-A71 test, including those who were never tested, those who had negative test results, and those who had positive test results for other viruses.

† Timing calculated among cases with the prodromal illness/symptom and documented dates of onset.

$\S$ Among 210 cases with CSF results available. Median cells $/ \mathrm{mm}^{3}$ calculated among cases with CSF pleocytosis ( $>5$ white blood cells per mm ${ }^{3}$ ).

" Supratentorial, brainstem, and cerebellar lesions among 227 cases with brain MRI reports available. Cervical cord lesions among 233 cases with cervical spine reports available, thoracic cord lesions among 205 cases with thoracic spine reports available, and conus lesions among 177 cases with lumbosacral spine reports available. Nerve root enhancement among 227 cases with a contrast MRI of the spine.

These findings also indicate that clinical characteristics of AFM patients might differ by viral etiology. However, these results should be interpreted with caution because 11 of the 12 EV-A71 cases were reported from a single state (Colorado), potentially influencing the description of EV-A71 cases. In addition, these results could be affected by biased ascertainment of viral infection if cases with certain characteristics (e.g., severe respiratory symptoms) were more likely to be tested early in the course of illness, when testing is more likely to yield a pathogen $(4,11)$. Some actual EV-D68 and EV-A71 cases likely did not have virus detected and were misclassified in the "other" category. However, these findings are consistent with other comparisons of EV-D68- and EV-A71-associated AFM cases, including those that compared cases within the same institution (18). Although there is considerable overlap in symptoms and findings associated with these two viruses, different viruses are likely associated with different AFM phenotypes.

Regardless of etiology, patients generally sought medical attention soon after onset of limb weakness; $76 \%$ of those with onset as an outpatient sought medical care within 1 day of onset of weakness, and most were initially evaluated in an emergency department. Because AFM can progress rapidly and lead to respiratory failure requiring intubation and mechanical ventilation, patients with suspected AFM should be immediately hospitalized and monitored for respiratory deterioration. Hospitalization also facilitates evaluation, including consultation with specialists and MRI of the brain and spine. Most patients were hospitalized within 1 day of onset of weakness, but $10 \%$ were not hospitalized until $\geq 4$ days after onset of limb weakness, perhaps indicating delays in recognition and an opportunity for improvement.

The findings in the report are subject to at least two limitations. First, this study was restricted to cases reported to CDC, which likely underestimate the actual number of AFM cases owing to underascertainment of AFM and underreporting of cases to health departments. Second, the analysis was limited to medical record abstraction data and, in many cases, to data from the early course of hospitalization. Although data on longterm outcomes were not available for this analysis, these data are now being collected and will be the subject of future reports.

Despite these limitations, the data in this report further elucidate the clinical characteristics of AFM and should aid recognition of signs and symptoms, subsequent evaluation, and referral to care. Early recognition of AFM is important for clinical management and for specimen collection and detection of the underlying etiology. AFM should be suspected in any child with acute flaccid limb weakness. Onset during the months of August-November of peak years, history of recent febrile respiratory illness, and presence of neck or back pain or any neurologic symptom should raise suspicion for AFM.

Based on recent trends, another peak AFM year is anticipated in 2020. It is not known whether or how the COVID-19 pandemic and recommended social distancing measures will affect enterovirus circulation or trends in AFM. COVID-19's impact on the health care system and health care-seeking behaviors will likely present additional challenges to the recognition and 
TABLE 3. Characteristics of hospitalization, treatment, and first medical encounter after onset of limb weakness among patients with confirmed acute flaccid myelitis ( $\mathrm{N}=238)$, by virus type - United States, 2018

\begin{tabular}{|c|c|c|c|c|}
\hline \multirow[b]{2}{*}{ Characteristic } & \multicolumn{4}{|c|}{ No. (\%) } \\
\hline & EV-D68 ( $=34)$ & EV-A71 ( $=12)$ & Other $(\mathrm{N}=192)^{*}$ & Total $(\mathrm{N}=238)$ \\
\hline \multicolumn{5}{|l|}{ Hospitalization } \\
\hline Hospitalized & $34(100)$ & $12(100)$ & $187(97)$ & $233(98)$ \\
\hline Hospitalized $\geq 1$ day before onset of limb weakness & $7(21)$ & $4(33)$ & $14(7)$ & $25(11)$ \\
\hline Hospitalized on same day or after onset of limb weakness & $27(79)$ & $8(67)$ & $171(89)$ & $206(87)$ \\
\hline Hospitalized, unknown timing & $0(一)$ & $0(-)$ & $2(1)$ & $2(1)$ \\
\hline \multicolumn{5}{|l|}{ Timing from onset of weakness to hospitalization, ${ }^{\dagger}$ days } \\
\hline Median interval from onset of weakness to hospitalization & 1 & 0.5 & 1 & 1 \\
\hline Range & $0-5$ & $0-5$ & $0-54$ & $0-54$ \\
\hline IQR & $0-2$ & $0-2.5$ & $0-2$ & $0-2$ \\
\hline $0-1$ & 20/27 (74) & $5 / 8(63)$ & 109/171 (64) & 134/206 (65) \\
\hline $2-3$ & $6 / 27(22)$ & $2 / 8(25)$ & 44/171 (26) & $52 / 206(25)$ \\
\hline $4-7$ & $1 / 27(4)$ & $1 / 8(13)$ & 8/171 (5) & 10/206 (5) \\
\hline$>7$ & $0 / 27(-)$ & $0 / 8(-)$ & 10/171 (6) & $10 / 206(5)$ \\
\hline \multicolumn{5}{|l|}{ Treatment received } \\
\hline Steroids, no IVIG & $8(24)$ & $1(8)$ & $46(24)$ & $55(23)$ \\
\hline IVIG, no steroids & $9(26)$ & $8(67)$ & $37(19)$ & $54(23)$ \\
\hline Both steroids and IVIG & $12(35)$ & $2(17)$ & $67(35)$ & $81(34)$ \\
\hline Plasma exchange & $5(15)$ & $1(8)$ & $26(14)$ & $32(13)$ \\
\hline Admitted to ICU & $25(74)$ & $5(42)$ & $99(52)$ & $129(54)$ \\
\hline Respiratory support & $19(56)$ & $3(25)$ & $43(22)$ & $65(27)$ \\
\hline Mechanical ventilation & $15(44)$ & $1(8)$ & $39(20)$ & $55(23)$ \\
\hline \multicolumn{5}{|l|}{ Location of first medical encounter after onset of weakness ${ }^{\S}$} \\
\hline Emergency department & $17 / 27(63)$ & $2 / 8(25)$ & 115/176 (65) & 134/211 (64) \\
\hline Primary care provider & $5 / 27(19)$ & $4 / 8(50)$ & 40/176 (23) & 49/211 (23) \\
\hline Urgent care provider & 4/27 (15) & $0 / 8(-)$ & 12/176 (7) & 16/211 (8) \\
\hline Unknown/missing/other & $1 / 27(4)$ & $2 / 8(25)$ & 9/176 (5) & $12 / 211(6)$ \\
\hline \multicolumn{5}{|l|}{ Timing from onset of limb weakness to first medical encounter, ${ }^{\S}$ days } \\
\hline Median interval from onset of weakness to first medical encounter & 0 & 0 & 1 & 0 \\
\hline Range & $0-3$ & $0-1$ & $0-15$ & $0-15$ \\
\hline $\mathrm{IQR}$ & $0-1$ & $0-0.5$ & $0-1$ & $0-1$ \\
\hline $0-1$ & $19 / 27(70)$ & $8 / 8(100)$ & $133 / 176(76)$ & $160 / 211(76)$ \\
\hline $2-3$ & $5 / 27(19)$ & $0 / 8(-)$ & 29/176 (16) & $34 / 211(16)$ \\
\hline $4-7$ & $0 / 27(-)$ & $0 / 8(-)$ & 4/176 (2) & 4/211 (2) \\
\hline$>7$ & $0 / 27(-)$ & $0 / 8(-)$ & $2 / 176(1)$ & 2/211 (1) \\
\hline Unknown/Missing & $3 / 27(11)$ & $0 / 8(-)$ & $8 / 176(5)$ & $11 / 211(5)$ \\
\hline
\end{tabular}

Abbreviations: $\mathrm{EV}$ = enterovirus; ICU = intensive care unit; IQR = interquartile range; IVIG = intravenous immunoglobulin.

* Other category includes all patients who did not have positive EV-D68 or EV-A71 test results, including those who were never tested, those who had negative test results, and those who had positive test results for other viruses.

${ }^{\dagger}$ Among the 206 patients (EV-D68 = 27; EV-A71 = 8; other $=171$ ) who were hospitalized on or after the date of onset of limb weakness.

$\S$ Among the 211 patients who had onset of limb weakness as an outpatient (206 patients who were hospitalized on or after the date of onset of limb weakness and five patients who were never hospitalized).

I Among 200 patients who had onset of limb weakness as an outpatient and known timing of onset of limb weakness and first medical encounter (211 patients minus the 11 with timing unknown).

evaluation of patients with AFM. Non-COVID-19 emergency department visits declined in 2020 (20), and the pandemic could possibly contribute to delays in care or to an increased proportion of clinical evaluations taking place via telephone or telemedicine. During this time, it will be critical for parents and clinicians to be aware of signs and symptoms suggestive of AFM and maintain vigilance for this condition during 2020.

\section{Acknowledgments}

Local and state health department Vaccine Preventable Diseases Surveillance coordinators; Grace Gombolay, Sarah Hopkins, Catherine Otten, Daniel Pastula, case review and classification neurology panel; Kiantra Butler, Brian Emery, Angela Guo, Adria Lee, Mona Marin, Manisha Patel, other participants, 2018 AFM response, CDC; CDC EIS officers and fellows who assisted with medical record abstraction.

Corresponding author: Sarah Kidd, skidd@cdc.gov, 404-639-8314.

${ }^{1}$ Division of Viral Diseases, National Center for Immunization and Respiratory Diseases, CDC; ${ }^{2}$ Division of STD Prevention, National Center for HIV/AIDS Viral Hepatitis, STD, and TB Prevention, CDC; ${ }^{3}$ Division of Global HIV and TB, Center for Global Health, CDC. 
All authors have completed and submitted the International Committee of Medical Journal Editors form for disclosure of potential conflicts of interest. W. Allan Nix and M. Steven Oberste have been issued U.S. patent numbers 7,247,457 and 7,714,122 for kits including VP1 and VP3 nucleic acid molecules. W. Allan Nix has been issued U.S. patent number 8,048,639 for detection and identification of parechoviruses. No other potential conflicts of interest were disclosed.

\section{References}

1. Van Haren K, Ayscue P, Waubant E, et al. Acute flaccid myelitis of unknown etiology in California, 2012-2015. JAMA 2015;314:2663-71. https://doi.org/10.1001/jama.2015.17275

2. Ayscue P, Van Haren K, Sheriff H, et al. Acute flaccid paralysis with anterior myelitis-California, June 2012-June 2014. MMWR Morb Mortal Wkly Rep 2014;63:903-6.

3. Pastula DM, Aliabadi N, Haynes AK, et al. Acute neurologic illness of unknown etiology in children-Colorado, August-September 2014. MMWR Morb Mortal Wkly Rep 2014;63:901-2.

4. Lopez A, Lee A, Guo A, et al. Vital signs: surveillance for acute flaccid myelitis-United States, 2018. MMWR Morb Mortal Wkly Rep 2019;68:608-14. https://doi.org/10.15585/mmwr.mm6827e1

5. Cortese MM, Kambhampati AK, Schuster JE, et al. A ten-year retrospective evaluation of acute flaccid myelitis at 5 pediatric centers in the United States, 2005-2014. PLoS One 2020;15:e0228671. https:// doi.org/10.1371/journal.pone.0228671

6. Uprety P, Curtis D, Elkan M, et al. Association of enterovirus D68 with acute flaccid myelitis, Philadelphia, Pennsylvania, USA, 20092018. Emerg Infect Dis 2019;25:1676-82. https://doi.org/10.3201/ eid2509.190468

7. Bitnun A, Yeh EA. Acute flaccid paralysis and enteroviral infections. Curr Infect Dis Rep 2018;20:34. https://doi.org/10.1007/s11908-018-0641-x

8. Sejvar JJ, Bode AV, Marfin AA, et al. West Nile virus-associated flaccid paralysis. Emerg Infect Dis 2005;11:1021-7. https://doi.org/10.3201/ eid1107.040991

9. Solomon T, Willison H. Infectious causes of acute flaccid paralysis. Curr Opin Infect Dis 2003;16:375-81. https://doi. org/10.1097/00001432-200310000-00002
10. Ooi MH, Wong SC, Clear D, et al. Adenovirus type 21-associated acute flaccid paralysis during an outbreak of hand-foot-and-mouth disease in Sarawak, Malaysia. Clin Infect Dis 2003;36:550-9. https:// doi.org/10.1086/367648

11. Sejvar JJ, Lopez AS, Cortese MM, et al. Acute flaccid myelitis in the United States, August-December 2014: results of nationwide surveillance. Clin Infect Dis 2016;63:737-45. https://doi.org/10.1093/ $\mathrm{cid} / \mathrm{ciw} 372$

12. Ayers T, Lopez A, Lee A, et al. Acute flaccid myelitis in the United States: 2015-2017. Pediatrics 2019;144:e20191619. https://doi.org/10.1542/ peds.2019-1619

13. Hixon AM, Yu G, Leser JS, et al. A mouse model of paralytic myelitis caused by enterovirus D68. PLoS Pathog 2017;13:e1006199. https:// doi.org/10.1371/journal.ppat.1006199

14. Sun S, Bian L, Gao F, et al. A neonatal mouse model of enterovirus D68 infection induces both interstitial pneumonia and acute flaccid myelitis. Antiviral Res 2019;161:108-15. https://doi.org/10.1016/j. antiviral.2018.11.013

15. Messacar K, Asturias EJ, Hixon AM, et al. Enterovirus D68 and acute flaccid myelitis-evaluating the evidence for causality. Lancet Infect Dis 2018;18:e239-47. https://doi.org/10.1016/S1473-3099(18)30094-X

16. Mishra N, Ng TFF, Marine RL, et al. Antibodies to enteroviruses in cerebrospinal fluid of patients with acute flaccid myelitis. MBio 2019;10:e01903-19. https://doi.org/10.1128/mBio.01903-19

17. Schubert RD, Hawes IA, Ramachandran PS, et al. Pan-viral serology implicates enteroviruses in acute flaccid myelitis. Nat Med 2019;25:1748-52. https://doi.org/10.1038/s41591-019-0613-1

18. Messacar K, Spence-Davizon E, Osborne C, et al. Clinical characteristics of enterovirus A71 neurological disease during an outbreak in children in Colorado, USA, in 2018: an observational cohort study. Lancet Infect Dis 2020;20:230-9. https://doi.org/10.1016/S1473-3099(19)30632-2

19. McLaren N, Lopez A, Kidd S, et al. Characteristics of patients with acute flaccid myelitis, United States, 2015-2018. Emerg Infect Dis 2020;26:212-9. https://doi.org/10.3201/eid2602.191453

20. Hartnett KP, Kite-Powell A, DeVies J, et al.; National Syndromic Surveillance Program Community of Practice. Impact of COVID-19 pandemic on emergency department visits-United States, January 1, 2019-May 30, 2020. MMWR Morb Mortal Wkly Rep 2020;69:699-704. https://doi.org/10.15585/mmwr.mm6923e1 